\title{
COMUNICAÇÃO
}

\section{AVALIAÇÃO DA UNIFORMIDADE DE APLICAÇÃO DE ÁGUA EM UM SISTEMA DE GOTEJAMENTO PARA PEQUENAS PROPRIEDADES ${ }^{1}$}

\author{
Uniformity of water application for a drip irrigation system to small farms \\ João Marcelo Silva do Nascimento², Luiz Antônio Lima ${ }^{3}$, Denis Cesar Cararo³, \\ Elvis Márcio Castro ${ }^{4}$, Marcus Vinicius Gama Silva ${ }^{4}$
}

\begin{abstract}
RESUMO
O uso de sistemas de irrigação por gotejamento à baixa pressão tem como objetivo oferecer uma técnica de irrigação viável para pequenas propriedades. Foram dimensionados oito sistemas de irrigação, um com os seguintes acessórios: 210 metros de tubo gotejador com diâmetro interno (DI) de 10,4 mm, sete conectores de $12 \mathrm{~mm}$, e seis metros de tubo de polietileno, um adaptador para tubo de polietileno, uma curva de $90^{\circ}$ e três metros de tubo de PVC com DI igual a 20,6 mm. Quatro sistemas foram montados com um filtro de tela de $130 \mu \mathrm{m}$ e os demais com um filtro de disco de $200 \mu \mathrm{m}$. Em todos os sistemas foram utilizados um reservatório de $1 \mathrm{~m}^{3}$ localizado a $3 \mathrm{~m}$ acima do nível do solo. Irrigou-se $1296 \mathrm{~m}^{2}$ de uma cultura de tomate, em canteiros com dimensões de 1,20 m por $60 \mathrm{~m}$. Para avaliação técnica do projeto foi obtida a uniformidade de aplicação de água através do coeficiente de uniformidade de emissão (UE) e coeficiente de uniformidade de distribuição (UD), durante um período de 90 dias. Os resultados indicaram que o UE e o UD foram excelentes e que os valores de grau de entupimento aumentaram com o uso da irrigação. Assim, de acordo com os resultados, comprova-se a viabilidade técnica da utilização de sistemas de irrigação por gotejamento funcionando a baixa pressão para pequenas áreas.
\end{abstract}

Termos para indexação: Irrigação localizada, distribuição de água, agricultura familiar, baixa pressão.

\begin{abstract}
A low pressure drip irrigation gravity feed system may be a viable technique to small farms. So, according with it, eight irrigation systems with $10.4 \mathrm{~mm}$ internal diameter driptubing $210 \mathrm{~m}$ long, seven $12 \mathrm{~mm}$ adapters, $20.6 \mathrm{~mm}$ polyethylene pipe $6 \mathrm{~m}$ long, polyethylene adapter, $20.6 \mathrm{~mm} \mathrm{90}$ elbow, $20.6 \mathrm{~mm}$ PVC pipe $3 \mathrm{~m}$ long and $1 \mathrm{~m}^{3}$ water reservoir at $3 \mathrm{~m}$ height, were designed and assembled to irrigate $1.296 \mathrm{~m}^{2}$ with tomato cultivated in $0.8 \times 60 \mathrm{~m}$ beds. One $130 \mathrm{~mm}$ screen filter was used to four systems and one $200 \mathrm{~mm}$ disc filter for the other ones. Emission uniformity coefficients (UE) and distribution uniformity coefficients (UD) were obtained throughout 90 days. UE and UD were excellent and the degree of clogging at the emitters increased with time. Therefore, according to the results, the low pressure drip irrigation systems tested are technically viable to small areas.
\end{abstract}

Index terms: Drip irrigation, low pressure, smallholders, water distribution.

(Recebido em 18 de maio de 2006 e aprovado em 9 de julho de 2008)

Para um melhor desenvolvimento das atividades humanas ligadas ao uso racional da água é importante o desenvolvimento de sistemas de irrigação cada vez mais eficientes evitando o desperdício de água. A irrigação localizada apresenta-se em destaque. Seus emissores aplicam água em pequenas vazões a alta freqüência, produzindo pequena área molhada, e mantendo a umidade do solo sempre próxima à capacidade de campo (KELLER \& KARMELI, 1975). Outras vantagens consistem em permitir o uso da fertirrigação, promovendo um melhor aproveitamento de fertilizantes, e possibilitar o parcelamento da aplicação de adubos de acordo com a freqüência de irrigação (BERNARDO et al., 2005).

Com o propósito de avaliar o funcionamento ideal do sistema de irrigação, incluindo o de irrigação localizada, emprega-se um índice de avaliação denominado de uniformidade de aplicação de água. Esse índice caracteriza um sistema de irrigação em função da diferença de volume de água aplicado pelos emissores ao longo das linhas laterais. A uniformidade de aplicação de água tem efeito

\footnotetext{
${ }^{1}$ Parte da dissertação apresentada à Universidade Federal de Lavras/UFLA para obtenção do título de mestre

Engenheiro Agrícola, Mestrando em Irrigação e Drenagem - Departamento de Engenharia Rural - Centro Federal de Educação Tecnológica/CEFET - Br 364, Km 329 - Vila de São Vicente - 78106-970 - Santo Antônio do Leverger, MT - jmarceloeagricola@yahoo.com.br

${ }^{3}$ Doutor, Professor Adjunto - Departamento de Engenharia/DEG - Universidade Federal de Lavras/UFLA - Cx. P. 3037 - $37200-000$ - Lavras, MG lalima@ufla.br; deniscesar@yahoo.com.br

${ }^{4}$ Graduandos em Engenharia Agrícola - Universidade Federal de Lavras/UFLA - Cx. P. 3037 - 37200-000 - Lavras, MG - elviscastrolima@yahoo.com.br; marquinho_gama@yahoo.com.br
} 
no rendimento de culturas e é considerada como um dos fatores mais importantes no dimensionamento e operação em sistemas de irrigação (BARRETO FILHO et al., 2000).

Para não prejudicar a uniformidade de aplicação de água, Keller \& Karmeli (1975) e Merriam \& Keller (1978) recomendam que a variação da vazão máxima dos gotejadores seja de $10 \%$ em todo o sistema de irrigação. De acordo com Bernardo et al. (2005), quando se trata de irrigação complementar, pode-se admitir um máximo de $20 \%$ da vazão nominal do emissor, o que corresponde a uma perda de carga ao longo da linha lateral de aproximadamente $44 \%$ da pressão de serviço para emissores que trabalham em regime de fluxo turbulento.

Com o objetivo de se determinar a uniformidade de aplicação de água de um sistema de irrigação, recomendase selecionar um número determinado de plantas. Merriam \& Keller (1978) recomendam um número de 16 plantas. Para isso, pode-se seguir o seguinte critério: elegese a subunidade a ser estudada e dela selecionam-se 4 linhas laterais: a primeira linha do sistema, a segunda situada a $1 / 3$ da origem, a terceira situada a $2 / 3$ da origem e a última lateral. Em cada lateral selecionam-se 4 plantas utilizando-se o mesmo critério (primeira, 1/3, 2/3 e a última) e faz-se a medição da vazão fornecida a cada planta.

Para classificação da uniformidade de aplicação de água, utilizam-se coeficientes, destacando: coeficiente uniformidade de distribuição (UD), Keller \& Karmeli (1974) classifica os valores como excelente quando superiores a $90 \%$, boa no intervalo de $85 \%$ a $90 \%$, regular entre $70 \%$ e $80 \%$ e ruim quando inferiores a $70 \%$; coeficiente uniformidade de emissão (UE) a norma ASAE EP-405.1 (ASAE, 1993) recomenda que o intervalo típico esteja entre $90 \%$ e $95 \%$, considerando um relevo uniforme (declividade menor que $2 \%$ ), com emissores tipo pontual e um número de emissores por planta inferior a três.

É de extrema importância conhecer a qualidade da água de irrigação a ser utilizada em um projeto de gotejamento. Em geral, pode-se dizer que uma água com condutividade elétrica ou CE menor do que $0,7 \mathrm{dS} \mathrm{m}{ }^{-1}$ não apresenta nenhuma restrição para uso em fertirrigação. Já valores de CE acima de 3,0 $\mathrm{dS} \mathrm{m}^{-1}$ passam a apresentar certa restrição de uso, sendo desaconselhável o uso da água para a irrigação. Outro parâmetro importante a ser avaliado é o $\mathrm{pH}$, que deve encontrar-se na faixa de 6,5 a 8,4, para possibilitar o uso da fertirrigação (FOLEGATTI et al., 2001).

Já Bucks et al. (1979) recomendam uma classificação segundo o risco de entupimento no uso da água de irrigação, de acordo com o tipo de problema: físico no qual analisa a quantidade de sólidos suspensos onde valores menores que $50 \mathrm{mg} \mathrm{L}^{-1}$ não apresentam riscos ao entupimento, já valores entre $50 \mathrm{mg} \mathrm{L}^{-1}$ e $100 \mathrm{mg} \mathrm{L}^{-1}$ médio risco e valores acima de $100 \mathrm{mg} \mathrm{L}^{-1}$ severo risco; químicos avaliam que o $\mathrm{pH}$ sendo menor do que 7,0 é considerado como de baixo risco, entre 7,0 e 8,0 médio risco e maior do que 8,0 severo risco, segundo a quantidade de sólidos dissolvidos um valor menor que $500 \mathrm{mg} \mathrm{L}^{-1}$ é considerado de baixo risco, entre 500 e 2000 médio risco e acima de 2000 severo risco, fatores biológicos no qual utiliza-se como parâmetro a unidade formadora de colônia(UFC/ $\mathrm{ml}$ ) em que valores menores que 10000 são de baixo risco, valores entre 10000 e 50000 médio risco e valores maiores que 50000 severo risco.

Estudos realizados por Rav-Acha et al. (1995), analisando a qualidade da água de irrigação, verificaram que $2 \mathrm{mg} \mathrm{L}^{-1} \mathrm{de}_{2}$ ou $\mathrm{ClO}_{2}$ causaram rápida inativação de bactérias, mas, até concentrações de $20 \mathrm{mg} \mathrm{L}^{-1}$ não afetaram o número de células de algas, embora tenha reduzido sua capacidade de multiplicação e quantidade de clorofila.

Visando uma alternativa econômica para auxiliar o desenvolvimento da população do meio rural, organizações internacionais têm proporcionado a implantação de tecnologias de baixo custo. Entre essas tecnologias, os sistemas de irrigação têm apresentado aceitação por fazendeiros e trabalhadores locais, devido às suas vantagens de flexibilidade, de simplicidade, e de ausência de mão-de-obra especializada para a sua montagem (NGIGI et al., 2001).

É aconselhável utilizar um sistema de irrigação que possibilite uma satisfatória uniformidade de aplicação de água em culturas de alto valor econômico que seja empregado, de maneira simples, na agricultura familiar em pequenas áreas. Objetivou-se, neste trabalho, avaliar a uniformidade de aplicação de água de sistemas de irrigação localizada funcionando a baixa pressão, em uma pequena área cultivada com tomateiro.

O sistema de irrigação proposto, foi desenvolvido utilizando-se um tubo gotejador da marca Plastro ${ }^{\circledR}$ modelo Hydrogol 12/25/1, cujos gotejadores são inseridos a um espaçamento de $0,30 \mathrm{~m}$, no próprio processo de fabricação. Esses emissores são constituídos de pequenos labirintos para a passagem da água, conforme apresenta-se na Figura 1, e por isso trabalham em fluxo turbulento. Na pressão de funcionamento de $98 \mathrm{kPa}$, esses emissores fornecem uma vazão de $1,0 \mathrm{~L} \mathrm{~h}^{-1}$. 


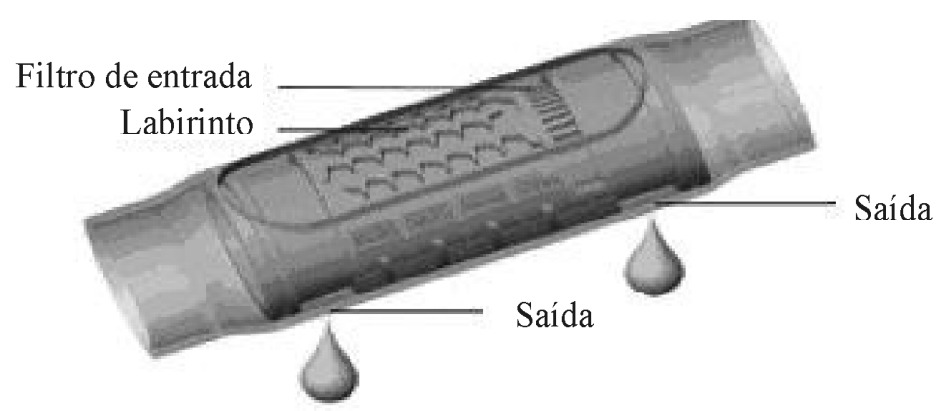

Figura 1 - Partes constituintes do gotejador da marca Plastro ${ }^{\circledR}$ modelo Hydrogol 12/25/1

Cada sistema de irrigação foi dimensionado considerando critérios internacionais, decidindo-se pela seguinte composição: 210 metros de tubo gotejador com diâmetro interno (DI) de 10,4 mm (sete linhas com condutos de $30 \mathrm{~m}$ ), filtros, sete conectores de $12 \mathrm{~mm}$, e $6 \mathrm{~m}$ de tubo de polietileno, adaptador para polietileno, curva de $90^{\circ}, 3 \mathrm{~m}$ de tubo de PVC com DI igual a 20,6 mm.

Foram utilizados dois reservatórios com capacidade de armazenamento de $1 \mathrm{~m}^{3}$ de água, que forneciam água a oito sistemas de irrigação. Na saída do primeiro, instalouse um filtro de tela com abertura de $130 \mu \mathrm{m}$ e, na saída do segundo instalou-se um filtro de disco com abertura de $200 \mu \mathrm{m}$.

Os oito sistemas de irrigação foram implantados em uma área experimental de $1296 \mathrm{~m}^{2}$, conforme apresentado na Figura 2, cuja propriedade pertence à UFLA/FAEPE e está localizada entre a cidade de Ijaci e o município de Macaia, estado de Minas Gerais. A área foi cultivada com plantas de tomate espaçadas de 0,6 por $0,8 \mathrm{~m}$, contendo dois gotejadores por planta, sobre canteiros com dimensões de 0,8 por $60 \mathrm{~m}$.

Após a implantação dos sistemas, iniciaram-se as irrigações com freqüência diária e com tempo de irrigação não superior a quatro horas, de acordo com as características de umidade do solo necessário para a formação de um bulbo úmido que satisfaça à demanda hídrica da cultura. No décimo dia foi efetuada a primeira avaliação da uniformidade de aplicação de água no sistema de irrigação.

No estudo da uniformidade de aplicação de água, para cada sistema foi calculado um índice definido por Keller \& Karmeli (1974) como uniformidade de distribuição, apresentado na Equação (1), e um índice, também definido pelos mesmos autores, como uniformidade de emissão, apresentado na Equação (2). O segundo caracteriza-se como mais rigoroso para o dimensionamento e avaliação em campo, pois possui as variações na uniformidade devido a fatores construtivos e fatores hidráulicos.
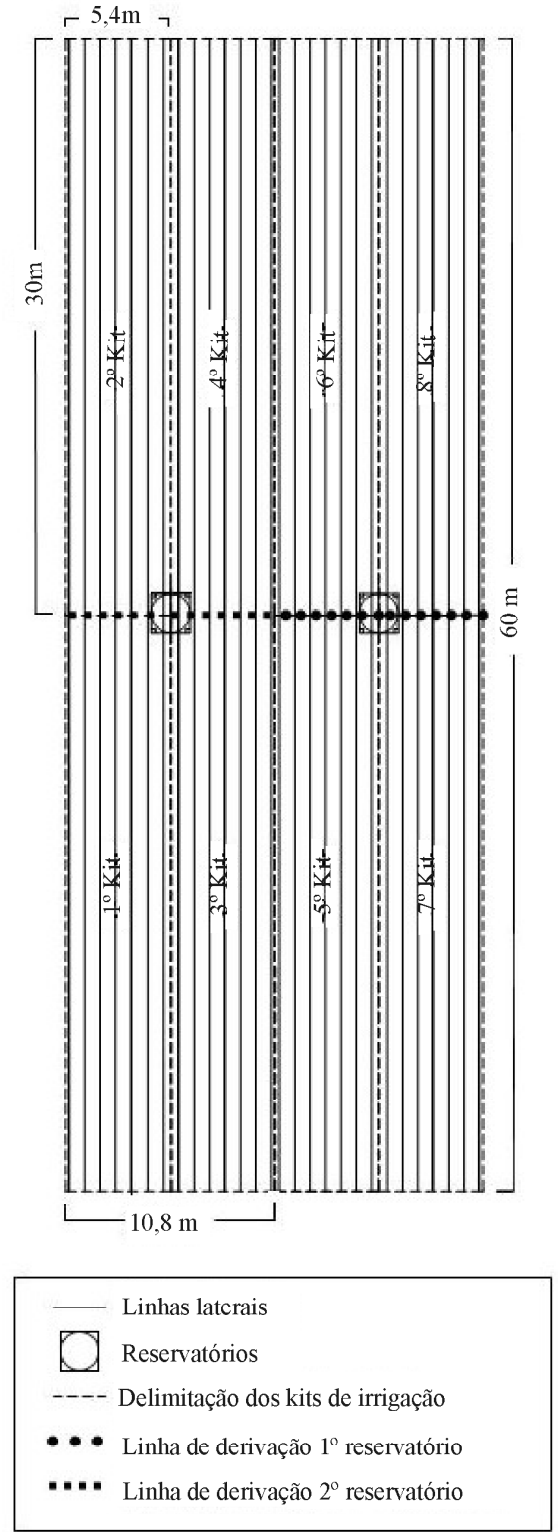

Figura 2 - Disposição dos sistemas de irrigação em campo 


$$
U D=\frac{q_{25}}{q_{m}} \cdot 100
$$

Equação 1

em que:

$U D=$ uniformidade de distribuição de água (\%);

$q_{25}=$ média das $25 \%$ das menores vazões $\left(\mathrm{L} \mathrm{h}^{-1}\right)$; e

$q_{m} \quad=$ vazão média do total de gotejadores $\left(\mathrm{L} \mathrm{h}^{-1}\right)$.

$$
U E=\left(1-\frac{1,27 \cdot C V f}{\sqrt{e}}\right) \cdot \frac{q_{n}}{q_{m}} \cdot 100
$$

Equação 2

em que:

$U E$ = uniformidade de emissão de água (\%);

$C V f=$ coeficiente de variação de fabricação (decimal);

$e \quad=$ número de emissores por planta; $\mathrm{e}$

$q_{n} \quad=$ vazão mínima $\left(\mathrm{L} \mathrm{h}^{-1}\right)$;

Para a caracterização da intensidade de obstrução nos emissores, avaliou-se a vazão dos últimos emissores dos oito sistemas de irrigação, e empregou-se a Equação (3) a cada sistema, que também foi utilizada por Cararo et al. (2006).

$$
G E=\left(1-\frac{q_{\text {usado }}}{q_{\text {novo }}}\right) \cdot 100
$$

Equação 3

em que:

$G E=$ grau de entupimento $(\%)$;

$q_{\text {usado }}=$ vazão do emissor usado $\left(\mathrm{L} \mathrm{h}^{-1}\right) ; \mathrm{e}$

$q_{\text {novo }}=$ vazão do emissor novo $\left(\mathrm{L} \mathrm{h}^{-1}\right)$.

Como o entupimento é proporcionalmente influenciado pela quantidade de agentes causadores de obstrução presentes no sistema, é imprescindível conhecer a qualidade da água de irrigação e efetuar a limpeza de filtros e linhas laterais. Foi realizada uma análise da condutividade elétrica, $\mathrm{pH}$, concentração de sólidos suspensos e sólidos dissolvidos da água no $57^{\circ}$ dia de irrigação, pelo Laboratório de Análise de Água do Departamento de Engenharia da UFLA. No $55^{\circ}$ dia de irrigação, depois de observada a tendência de redução dos valores de uniformidade de aplicação de água, a aparência visual da água e o grau de entupimento, decidiuse adicionar hipoclorito de sódio a uma concentração de $20 \mathrm{mg} \mathrm{L}^{-1}$ ao reservatório, conforme recomendado por RavAcha et al. (1995).

Durante o desenvolvimento da cultura, foram realizadas fertirrigações, considerando as características de compatibilidade e solubilidade dos fertilizantes, a qualidade da água, a limpeza dos filtros e a lavagem dos produtos químicos do interior das tubulações, visando o satisfatório desempenho dos sistemas de irrigação. Os fertilizantes foram dissolvidos nos próprios reservatórios de abastecimento, a cada aplicação, em períodos e quantidades pré-determinados pela demanda nutricional do tomateiro. Utilizaram-se $2000 \mathrm{mg} \mathrm{L}^{-1}$ da formulação contendo MAP (fosfato monoamônico), uréia e $\mathrm{KNO}_{3}$ semanalmente, $400 \mathrm{mg} \mathrm{L}^{-1}$ de adubo à base de micronutrientes e fósforo (Aminosan ${ }^{\circledR}$ ) a cada duas semanas, e uso intercalado de $400 \mathrm{mg} \mathrm{L}^{-1}$ de $\mathrm{CaNO}_{3}$. Para evitar a precipitação química pela reação do $\mathrm{CaNO}_{3}$ com a formulação contendo MAP, optou-se em aplicações, destes fertilizantes, em diferentes dias da semana.

Para caracterizar o tubo gotejador em baixa pressão, foi construída uma bancada de testes em laboratório, com um reservatório elevado a $3 \mathrm{~m}$, obtendo uma vazão média dos emissores de $0,43 \mathrm{~L} \mathrm{~h}^{-1}$, e um coeficiente de variação de fabricação de 2,59\% à pressão de 24,52 kPa no início da linha lateral, descontadas as perdas de carga no conduto.

Pela análise da água de irrigação, verificaram-se os seguintes resultados: $\mathrm{CE}$ igual a $0,17 \mathrm{dS} \mathrm{m}^{-1}$, não apresentando restrição para o uso da fertirrigação, segundo Folegatti (2001); pH igual a 7,8, classificado como médio risco ao entupimento, de acordo com Bucks et al. (1979); concentração de sólidos suspensos igual a $13 \mathrm{mg}$ $\mathrm{L}^{-1}$ e sólidos dissolvidos igual a $145 \mathrm{mg} \mathrm{L}^{-1}$, ambos de baixo risco ao entupimento segundo Bucks et al. (1979). Portanto, a água utilizada para as irrigações, em geral, foi considerada de boa qualidade.

O emprego de oito sistemas de irrigação permitiu uma maior confiabilidade nos resultados, devido a um maior número de emissores amostrados. Os valores de uniformidade de aplicação de água apresentam-se na Tabela 1.

Os valores de UD, observados na Tabela 1, variaram de $99 \%$ aos 10 dias de uso, no $7^{\circ}$ sistema, até $90,2 \%$ aos 90 dias de uso, no $1^{\circ}$ sistema. Assim, todos os sistemas de irrigação apresentaram excelente UD de acordo com a classificação proposta por Keller \& Karmeli (1974). Para a UE, os valores variaram de $95,4 \%$ aos 10 dias de uso, no $1^{\circ}$ e no $4^{\circ}$ sistema, até $85,2 \%$ aos 90 dias de uso, no $5^{\circ}$ sistema, permanecendo essas porcentagens, dentro do intervalo típico estipulado para uma satisfatória uniformidade de acordo com a norma ASAE (1993).

Apesar da excelente uniformidade observada na Tabela 1, nota-se que há uma tendência em decréscimo dos valores ao longo do tempo de uso dos sistemas. Isso pode ter como causas, a obstrução parcial por algas, bactérias e impurezas do fertilizante ou o desgaste físico 
dos emissores, pelo uso contínuo de produtos químicos. A tendência não foi interrompida, expressivamente, pela aplicação de hipoclorito de sódio próximo aos 50 dias, e mesmo as porcentagens de UE, cujo índice é mais rigoroso, continuaram a decrescer após os 70 dias. Esse fato indica que há necessidade de uma cloração freqüente para se obter valores maiores que os apresentados nos 90 dias.
O grau de entupimento é apresentado na Tabela 2. Observa-se um aumento gradativo no grau de entupimento dos últimos emissores com o passar dos dias de irrigação, desde valores de 2,3\% aos 10 dias de uso, no $1^{\circ}$ sistema de irrigação, até $21,0 \%$ aos 90 dias de uso, no $8^{\circ}$ sistema. Esse fato provavelmente ocasionou uma diminuição na uniformidade de aplicação de água, porém não inviabilizou a técnica de irrigação a baixa pressão, para pequenas áreas.

Tabela 1 - Uniformidade de distribuição (UD) e uniformidade de emissão (UE) para os sistemas de irrigação avaliados durante o período de uso

\begin{tabular}{ccccccccc}
\hline \multirow{2}{*}{ Dias de uso } & \multicolumn{7}{c}{ Sistemas de irrigação } \\
\cline { 2 - 9 } & 1 & 2 & 3 & 4 & 5 & 6 & 7 & 8 \\
\hline & & \multicolumn{7}{c}{ Uniformidade de distribuição - UD (\%) } \\
30 & 97,7 & 97,6 & 97,7 & 97,7 & 97,6 & 97,5 & 99,0 & 95,0 \\
50 & 97,6 & 95,2 & 95,4 & 95,2 & 95,0 & 95,0 & 95,0 & 95,0 \\
70 & 92,9 & 95,2 & 92,9 & 95,1 & 95,0 & 97,4 & 94,9 & 94,9 \\
90 & 92,7 & 95,1 & 95,1 & 92,7 & 92,5 & 94,9 & 94,7 & 92,3 \\
& 90,2 & 92,4 & 95,0 & 92,5 & 92,3 & 94,7 & 94,7 & 94,7 \\
10 & & & & & & & & \\
30 & 95,4 & 95,3 & 95,4 & 95,4 & 92,9 & 95,2 & 92,8 & 92,8 \\
50 & 93,0 & 93,0 & 93,0 & 93,0 & 92,9 & 92,8 & 87,9 & 87,9 \\
70 & 88,4 & 88,4 & 88,4 & 90,5 & 87,9 & 90,2 & 90,2 & 90,8 \\
90 & 92,8 & 90,5 & 90,5 & 85,8 & 87,9 & 90,2 & 92,5 & 85,2 \\
& 85,8 & 85,8 & 87,9 & 87,9 & 85,2 & 87,4 & 87,4 & 87,4 \\
\hline
\end{tabular}

Tabela 2 - Grau de entupimento (\%) dos últimos emissores de cada linha lateral, em estudo durante o período de uso do sistema de irrigação

\begin{tabular}{|c|c|c|c|c|c|c|c|c|c|}
\hline \multirow{2}{*}{ Dias de uso } & \multirow{2}{*}{ Lateral } & \multicolumn{8}{|c|}{ Sistemas de irrigação } \\
\hline & & $1^{\circ}$ & $2^{\circ}$ & $3^{\circ}$ & $4^{\circ}$ & $5^{\circ}$ & $6^{\circ}$ & $7^{\circ}$ & $8^{\circ}$ \\
\hline \multirow{4}{*}{10} & 1 & 2,3 & 4,6 & 2,3 & 2,3 & 7,0 & 7,0 & 7,0 & 11,6 \\
\hline & 3 & 2,3 & 4,6 & 2,3 & 2,3 & 11,6 & 7,0 & 11,6 & 7,0 \\
\hline & 5 & 2,3 & 4,6 & 2,3 & 2,3 & 7,0 & 7,0 & 11,6 & 11,6 \\
\hline & 7 & 2,3 & 4,6 & 2,3 & 2,3 & 11,6 & 11,6 & 11,6 & 11,6 \\
\hline \multirow{4}{*}{30} & 1 & 2,3 & 7,0 & 2,3 & 7,0 & 11,6 & 7,0 & 11,6 & 7,0 \\
\hline & 3 & 2,3 & 7,0 & 7,0 & 7,0 & 11,6 & 11,6 & 11,6 & 11,6 \\
\hline & 5 & 7,0 & 7,0 & 7,0 & 7,0 & 11,6 & 16,2 & 11,6 & 11,6 \\
\hline & 7 & 7,0 & 7,0 & 7,0 & 7,0 & 7,0 & 11,6 & 11,6 & 16,3 \\
\hline \multirow{4}{*}{50} & 1 & 7,0 & 7,0 & 7,0 & 7,0 & 7,0 & 7,0 & 11,6 & 11,6 \\
\hline & 3 & 7,0 & 11,6 & 7,0 & 7,0 & 11,6 & 11,6 & 11,6 & 16,3 \\
\hline & 5 & 11,6 & 7,0 & 11,6 & 11,6 & 11,6 & 16,3 & 16,3 & 11,6 \\
\hline & 7 & 11,6 & 7,0 & 11,6 & 11,6 & 11,6 & 16,3 & 11,6 & 16,3 \\
\hline
\end{tabular}

Continuação... 
Tabela 2 - Continuação...

\begin{tabular}{cccccccccc}
\hline \multirow{3}{*}{70} & 1 & 11,6 & 11,6 & 11,6 & 11,6 & 11,6 & 11,6 & 11,6 & 16,3 \\
& 3 & 11,6 & 11,6 & 7,0 & 7,0 & 16,3 & 16,3 & 16,3 & 11,6 \\
& 5 & 11,6 & 11,6 & 11,6 & 11,6 & 16,3 & 16,3 & 11,6 & 16,3 \\
& 7 & 11,6 & 11,6 & 11,6 & 16,3 & 11,6 & 16,3 & 16,3 & 16,3 \\
& 1 & 11,6 & 11,6 & 16,3 & 16,3 & 16,3 & 11,6 & 16,3 & 16,3 \\
90 & 3 & 16,3 & 11,6 & 11,6 & 11,6 & 16,3 & 20,93 & 16,3 & 11,6 \\
& 5 & 11,6 & 16,3 & 16,3 & 11,6 & 16,3 & 16,3 & 21,0 & 21,0 \\
& & 16,3 & 11,6 & 7,0 & 16,3 & 20,93 & 16,3 & 16,3 & 21,0 \\
\hline
\end{tabular}

Analisando os resultados, conclui-se que os sistemas de irrigação apresentam bom desempenho hidráulico, contudo, assim como todo sistema de irrigação localizada, há necessidade que o irrigante tome alguns cuidados quanto à qualidade da água, à fertirrigação e à limpeza do sistema, com o propósito de garantir maior durabilidade do equipamento com satisfatória uniformidade de aplicação de água.

\section{AGRADECIMENTOS}

A Coordenação de Aperfeiçoamento de Pessoal de Nível Superior (CAPES), pelo apoio financeiro disponibilizado ao longo desta pesquisa.

A Plastro do Brasil, sediada em Uberlândia, Minas Gerais, pela doação dos materiais para a montagem dos ensaios laboratoriais e de campo.

A Hortiagro Sementes Ltda., através do Sr. Paulo Moretto e Vicente Licursi, da Fazenda Palmital, pela área cedida para a montagem do sistema de irrigação.

\section{REFERÊNCIAS BIBLIOGRÁFICAS}

ASAE. ASAE EP 405.1: standards, design and installation of microirrigation systems. [S.1.], 1993.

BARRETO FILHO, A. de A.; DANTAS NETO, J.; MATOS, J. A. de; GOMES, E. M.; Desempenho de um sistema de irrigação por microaspersão, instalado a nível de campo Revista Brasileira Engenharia Agrícola e Ambiental, Campina Grande, v. 4, n. 3, p. 309-314, 2000.

BERNARDO, S.; SOARES, A. A.; MANTOVANI, E. C. Manual de irrigação. 7. ed. Viçosa: UFV, 2005. 596 p.

BUCKS, D. A.; NAKAYAMA, F. S.; GILBERT, R. G. Trickle irrigation water quality and preventive maintenance. Agricultural and Water Management, Amsterdam, v. 2, p. 149-162, 1979.

CARARO, D. C.; BOTREL, T. A.; HILLS, D. J.; LEVERENZ, H. L. Analysis of clogging in drip emitters during wastewater irrigation. Applied Engineering in Agriculture, v. 22, n. 2, p. 251-257, 2006.

FOLEGATTI, M. V.; CASARINI, E.; BLANCO, F. F.; BRASIL, R. P. C. do; RESENDE, R. S. Fertirrigação: flores, frutas e hortaliças. Guaíba: Agropecuária, 2001. v. 2, $336 \mathrm{p}$.

KELLER, J.; KARMELI, D. Trickle irrigation design parameters. Transactions of the ASAE, Saint Joseph, v. 17, n. 4, p. 678-684, 1974.

KELLER, J.; KARMELI, D. Trickle irrigation design. Glendora: Rain Bird Sprinkle Manufacturing, 1975.

MERRIAM, J. L.; KELLER, J. Farm irrigation system evaluation: a guide for management. Logan: Utah State University, 1978.

NGIGI, S. N.; WAWERU, J. N.; BLANK, H. G. Low-cost irrigation for poverty reduction. 2001. Disponível em: $<\mathrm{http}: / /$ www.iwmi.cgiar.org/pubs/Areps/2001/ 'theme $\% 202$.pdfi $>$. Acesso em: 14 out. 2005.

RAV-ACHA, C.; KUMMEL, M.; SALAMON, I.; ADIN, A. The effect of chemical oxidants on effluent constitutents for drip irrigation. Water Research, v. 29, n. 1, p. 119-129, Jan. 1995. 